\title{
Deletion of TLR5 results in spontaneous colitis in mice
}

\author{
Matam Vijay-Kumar, ${ }^{1}$ Catherine J. Sanders, ${ }^{1}$ Rebekah T. Taylor, ${ }^{1}$ \\ Amrita Kumar, ${ }^{1}$ Jesse D. Aitken,, ${ }^{1}$ Shanthi V. Sitaraman, ${ }^{2}$ Andrew S. Neish, ${ }^{1}$ \\ Satoshi Uematsu, ${ }^{3}$ Shizuo Akira, ${ }^{3}$ Ifor R. Williams, ${ }^{1}$ and Andrew T. Gewirtz ${ }^{1}$ \\ 1Department of Pathology and 2Department of Medicine, Emory University, Atlanta, Georgia, USA. \\ ${ }^{3}$ Department of Host Defense, Research Institute for Microbial Diseases, Suita, Osaka, Japan.
}

\begin{abstract}
Activation of TLRs by bacterial products results in rapid activation of genes encoding products designed to protect the host from perturbing microbes. In the intestine, which is colonized by a large and diverse population of commensal bacteria, TLR signaling may not function in a simple on/off mode. Here, we show that the flagellin receptor TLR5 has an essential and nonredundant role in protecting the gut from enteric microbes. Mice lacking TLR5 (TLR5KO mice) developed spontaneous colitis, as assessed by well-defined clinical, serologic, and histopathologic indicators of this disorder. Compared with WT littermates, TLR5KO mice that had not yet developed robust colitis exhibited decreased intestinal expression of TLR5-regulated host defense genes despite having an increased bacterial burden in the colon. In contrast, such TLR5KO mice displayed markedly increased colonic expression of hematopoietic-derived proinflammatory cytokines, suggesting that elevated levels of bacterial products may result in activation of other TLRs that drive colitis in TLR5 KO mice. In accordance, deletion of TLR4 rescued the colitis of TLR5 KO mice in that mice lacking both TLR4 and TLR5 also had elevated bacterial loads in the colon but lacked immunological, histopathological, and clinical evidence of colitis. That an engineered innate immune deficiency ultimately results in spontaneous intestinal inflammation supports the notion that an innate immune deficiency might underlie some instances of inflammatory bowel disease.
\end{abstract}

\section{Introduction}

Detection of bacterial products by germline-encoded receptors of the innate immune system, particularly TLRs, results in rapid activation of gene expression designed to protect the host from the perturbing microbe(s). Genes whose expression is activated by TLR signaling include those with direct antibacterial actions and cytokines that drive recruitment and/or activation of immune cells. The immune cell, especially neutrophil, recruitment to mucosal surfaces that results from such chemokine expression is thought to play an important role in protecting against bacterial dissemination but may also underlie the clinical manifestations associated with inflammation as well as the tissue damage therein. In the intestine, which is normally colonized by a large and diverse population of commensal bacteria, TLR signaling may not function in a simple on/off mode but rather also appears to play a role in homeostasis (1), possibly via specialized pools of TLR2 or TLR9 located on the apical surface of the intestinal epithelium $(2,3)$. Mice with a broad deficiency in TLR signaling due to loss of the signaling adaptor MyD88 do not display an overt intestinal phenotype but show clear phenotypic differences in models of colitis. Specifically, MyD88KO mice develop exacerbated injury upon exposure to dextran sodium sulfate (1). In contrast, deficiency in MyD88 protects mice against the spontaneous colitis that occurs in mice lacking the gene for IL-10 (4). Together, these results indicate that TLR signaling in general can drive intestinal inflamma-

Nonstandard abbreviations used: Ang4, angiogenin-4; DKO, double KO; KC, keratinocyte-derived chemokine; MPO, myeloperoxidase; SAA, serum amyloid A; T5KO-H, TLR5KO with high SAA levels; T5KO-L, TLR5KO with low SAA levels.

Conflict of interest: The authors have declared that no conflict of interest exists.

Citation for this article: J. Clin. Invest. 117:3909-3921 (2007). doi:10.1172/JCI33084. tion upon immune dysregulation but may normally serve to protect the gut from some challenges.

While the intestinal commensal microflora are a potentially large source of various TLR ligands, a number of observations suggest a particularly important role for the TLR5 ligand flagellin. Gut epithelial cells (by far the most numerous cell type in the intestine) derived from a number of sources (i.e., cell lines) uniformly exhibit robust proinflammatory gene expression in response to flagellin while such cells have mostly been observed to be relatively hyporesponsive to the quintessential TLR agonist $\operatorname{LPS}(5,6)$ and respond nonclassically to TLR2 ligands (2). Flagellin has been suggested to be a major target of innate and adaptive immunity in Crohn disease and, in some ethnicities, heterozygous carriage of a dominant negative TLR5 allele is associated with protection from Crohn disease (7-9). Consequently, we hypothesized that mice lacking the flagellin receptor TLR5 might exhibit a reduced tendency to develop intestinal inflammation. Rather, we observed that loss of TLR5-mediated gene expression resulted in inability of mice to manage their commensal microflora and development of spontaneous colitis. These results indicate TLR5 plays an essential role in protecting the intestine from normally commensal bacteria.

\section{Results}

TLR5KO mice develop spontaneous colitis. Mice with a targeted deletion in TLR5 were generated on a mixed C57BL/6 × 129/Sv background at the Research Institute for Microbial Diseases, Suita, Osaka, Japan (10). Such mice appeared generally healthy, and study of the small intestine did not reveal gross phenotypic alterations. Mice that were heterozygous-null for TLR5 underwent quarantine at Charles River Laboratories, were transported to Emory University for further backcrossing to C57BL/6, and 
A

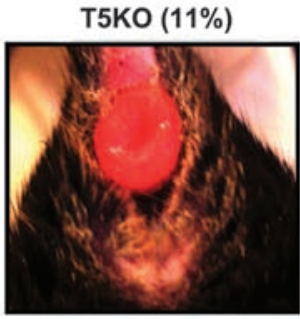

B

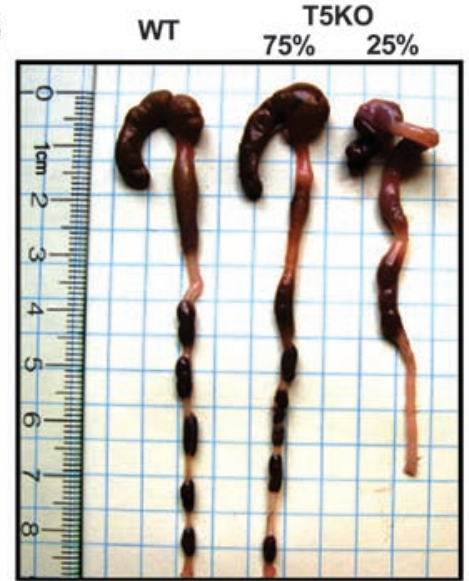

C

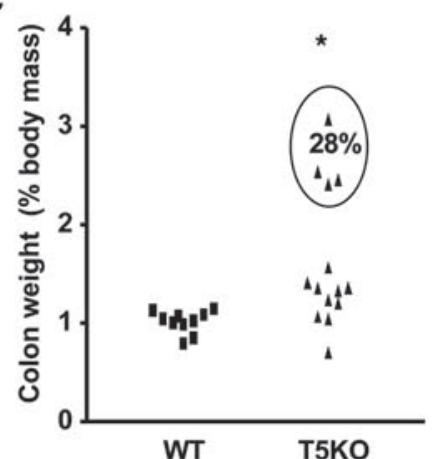

D

Colon

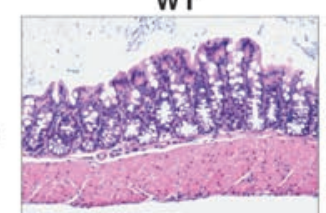

Cecum

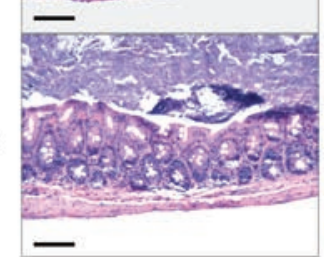

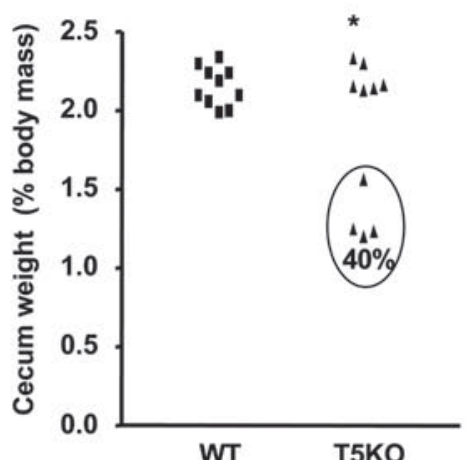

E

T5KO
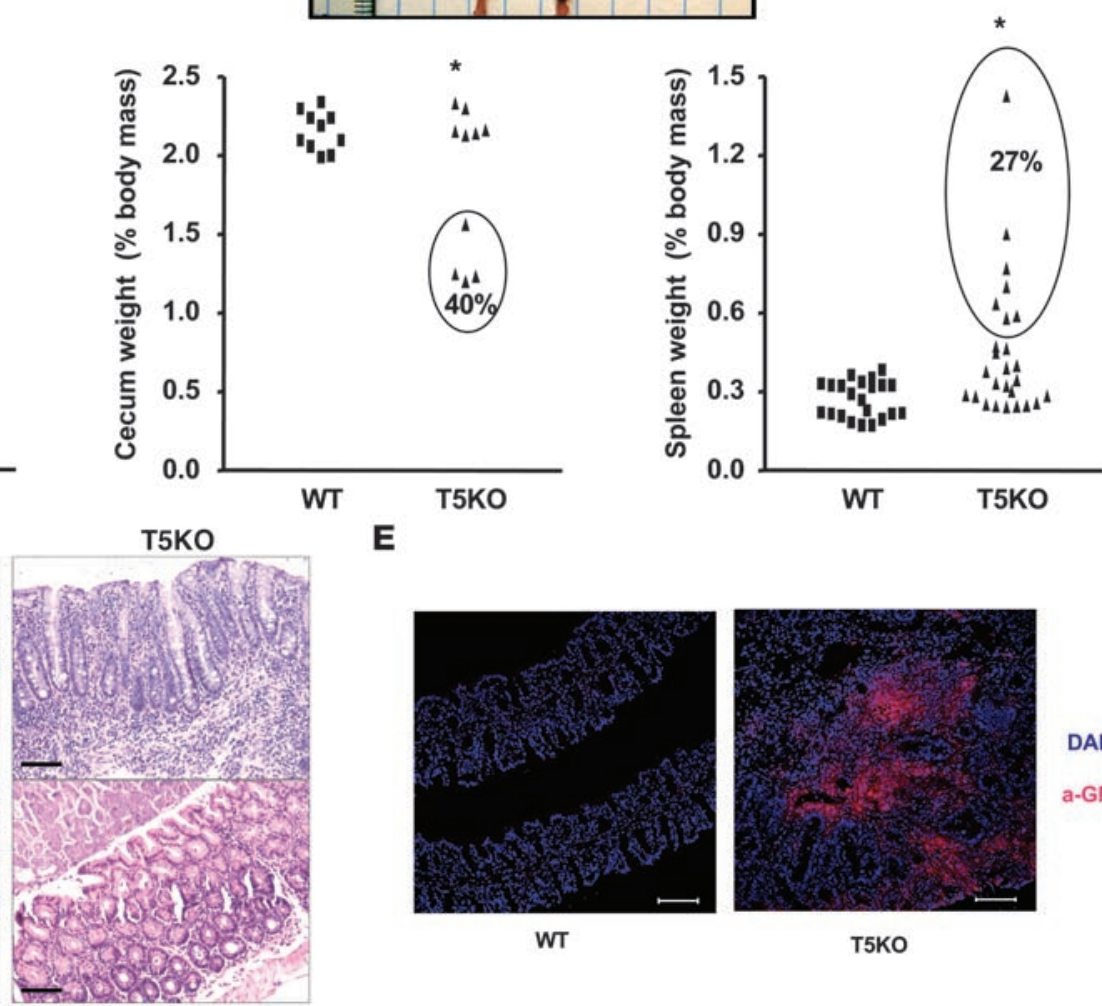

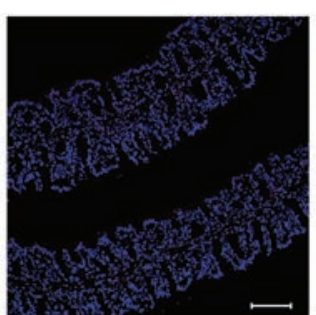

WT

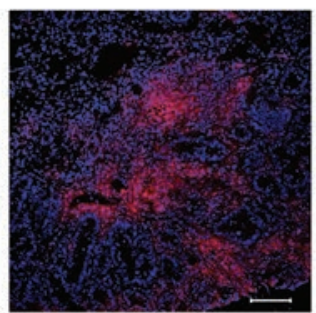

T5KO

Figure 1

TLR5KO mice develop spontaneous colitis. (A) Representative rectal prolapse exhibited by about 10\% of TLR5KO (T5KO) mice. (B) Representative gross appearance of cecum/colon of TLR5KO mice and WT littermates. (C) Comparison of organ weights from TLR5KO mice and WT littermates. (D) H\&E-stained sections of cecum and proximal colon of TLR5KO mice and WT littermates. Note the extensive immune cell infiltrates, focal crypt epithelial destruction, edema, and epithelial hyperplasia in TLR5KO mice. (E) Immunofluorescent micrographs showing neutrophils stained with anti-GR1 (in red) and counterstained with DAPI to show all nuclei in blue. Scale bars: $100 \mu \mathrm{M} .{ }^{*} P<0.05$.

were then bred to make colonies of mice homozygous-null for TLR5 (TLR5KO) and WT littermates. That absence of both copies of the TLR5 gene resulted in loss of TLR5 function was verified by observing loss of serum cytokines in response to systemically administered flagellin. Specifically, as expected, TLR5KO mice exhibited dramatically reduced induction of serum cytokines such as keratinocyte-derived chemokine (KC) (murine homolog of IL-8) and IL-6 in response to systemically administered purified flagellin (Supplemental Figure 1; supplemental material available online with this article; doi:10.1172/JCI33084DS1) in accordance with recent studies $(10,11)$. Interestingly, the level of $\mathrm{KC}$ induced by flagellin in C57BL/ 6 mice was higher than that induced by LPS, although flagellin, unlike LPS, failed to induce TNF- $\alpha$ (in WT or TLR5KO mice) (Supplemental Figure 2).

The majority of TLR5KO mice appeared to be generally healthy although they tended to be smaller than their age-matched, sexmatched WT littermates (body weights of 4-week-old females [g]: WT vs. KO, $16.3 \pm 0.38$ vs. $15.1 \pm 0.24$; males: WT vs. KO, $19.2 \pm 0.41$ vs. $17.6 \pm 0.57 ; P<0.05)$. However, the most striking phenotypic feature displayed by these mice was their frequency of rectal prolapse. Specifically, we observed that, at 3 levels of backcrossing (N4, N6, and N8), about $10 \%-12 \%$ mice lacking TLR5 but none of 
A

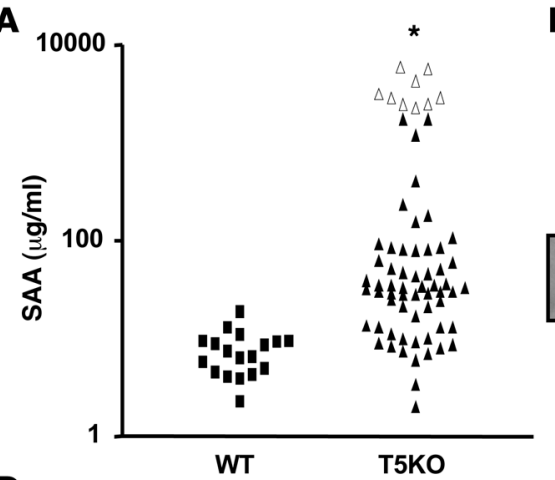

D

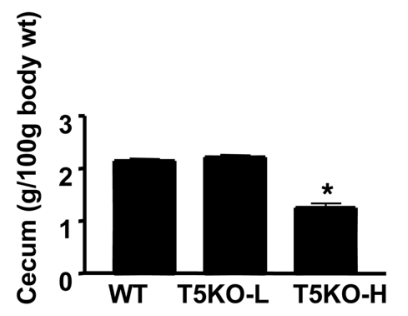

B
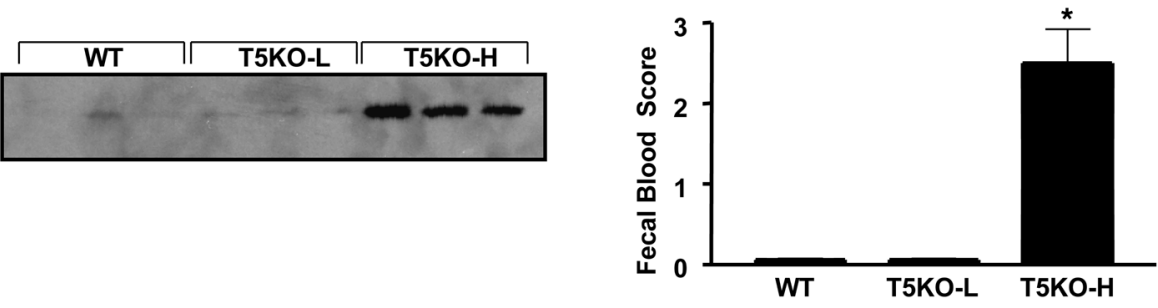

E
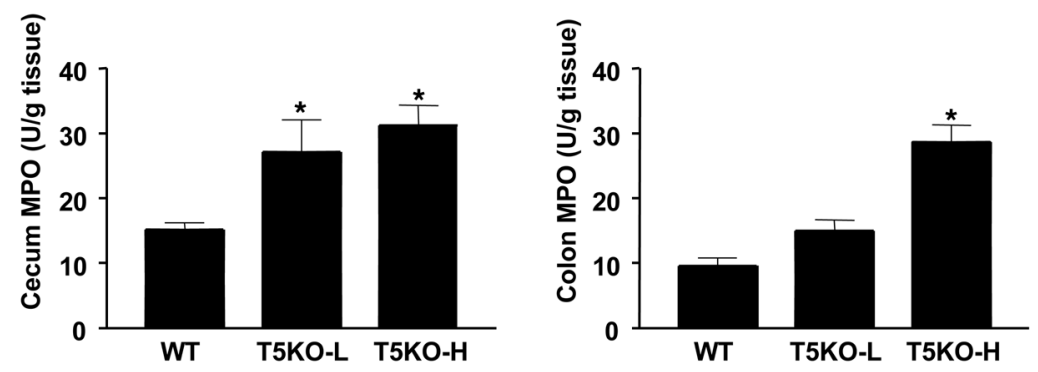

Figure 2

SAA levels mark severity of colitis in TLR5KO mice. Eight- to twelve-week-old TLR5KO mice and WT littermates were euthanized and parameters assayed. (A) SAA: open triangles represent mice with rectal prolapse at time of serum collection. SAA levels were then used to stratify mice for analysis in B-E. T5KO-H indicates SAA >100 $\mu \mathrm{g} / \mathrm{ml}$; T5KO-L indicates SAA< $50 \mu \mathrm{g} / \mathrm{ml}$. (B) Immunoblot showing serum lipocalin 2. (C) Occult blood in feces (D) Weights of cecum, colon, and spleen. (E) Cecum and colon MPO levels. ${ }^{*} P<0.05$, significant difference from WT mice.

their WT littermates exhibited spontaneous rectal prolapse. Interestingly, prolapse has not been observed in TLR5KO mice maintained in Japan. In contrast with TLR5KO mice, mice lacking other TLRs (i.e., 2, 3, 4, 11) and the global TLR-signaling adapter MyD88 bred in our facility have not exhibited this phenotype whereas IL-10-null mice bred in our facility develop prolapse at the previously observed frequency (12). Rectal prolapse in TLR5KO mice was seemingly irreversible and correlated with unsuccessful breeding. Nonetheless, continued breeding of nonprolapsed TLR5KO mice produced offspring that continued to prolapse with substantial frequency (40 of 365) while offspring of their littermates did not show this phenotype ( 0 of > 400) (Figure $1 \mathrm{~A}$ ).

Rectal prolapse and reduced body weights are hallmarks of spontaneous murine colitis, and therefore we next determined whether TLR5KO mice were developing colitis as assessed by several welldefined indicators of this disease state. Specifically, excluding TLR5KO mice that had prolapsed (which all had robust transmural colitis), we compared TLR5KO mice with their WT littermates in terms of intestinal bleeding, morphology, organ weights, and histopathology. Additionally, we measured body weight and weights of their cecum, colon, liver, and spleen. Approximately $20 \%$ of the nonprolapsed TLR5KO mice exhibited detectable spontane- ous bleeding (gross or occult), which was not detected in any WT mice. Moreover, 25\% of TLR5KO mice displayed gross features of robust colitis including contracted ceca, swollen, opaque proximal colon, and absence of well-formed stools (Figure 1B). Such appearances were not exhibited by any of the WT mice. These alterations in the appearance of cecum and colon correlated with and thus could be quantitated by simply measuring organ weights, which were significantly altered in about $30 \%$ of the TLR5KO mice that had not exhibited spontaneous rectal prolapse when euthanized (Figure 1C). Further consistent with the colitic state, TLR5KO mice exhibited substantially enlarged spleens. Splenomegaly closely correlated with substantially enlarged mesenteric and linguinal lymph nodes (data not shown). Histopathologic analysis confirmed the notion that a portion of TLR5KO mice had colitis (regardless of whether they had rectal prolapse). Specifically, in mice displaying gross alterations in intestinal appearance (or organ weights), both the cecum and proximal colon displayed extensive areas of epithelial hyperplasia associated with mononuclear infiltrates, focal crypt epithelial destruction, and edema (Figure 1D), which was not present in WT littermates or in the $60 \%$ of TLR5KO mice whose intestines appeared normal by the above parameters. In accordance with histological appearance, use of a neutrophil- 
Table 1

Histopathologic scoring of colitis

\begin{tabular}{|c|c|c|c|c|c|c|c|c|}
\hline \multirow[b]{2}{*}{ Strain } & \multicolumn{4}{|c|}{ Cecum } & \multicolumn{4}{|c|}{ Colon } \\
\hline & Infil. (0-3) & Ed. $(0-2)$ & CL (0-3) & $\mathrm{GD}(0-2)$ & Infil. (0-3) & Ed. $(0-2)$ & $C L(0-3)$ & GD (0-2) \\
\hline WT & 0 & $0.33 \pm 0.33$ & 0 & 0 & 0 & $0.33 \pm 0.33$ & 0 & 0 \\
\hline T5KO-L & $0.67 \pm 0.33$ & $0.33 \pm 0.33$ & 0 & 0 & $0.33 \pm 0.33$ & $0.67 \pm 0.33$ & 0 & 0 \\
\hline T5KO-H & $2.67 \pm 0.33$ & $1.0 \pm 0.57$ & $2.67 \pm 0.33$ & $1.67 \pm 0.33$ & 3 & $1.67 \pm 0.33$ & 3 & $2 \pm 0.33$ \\
\hline T4/5DKO & $0.67 \pm 0.33$ & $1.0 \pm 0.57$ & $0.33 \pm 0.33$ & 0 & $0.33 \pm 0.33$ & 0 & 0 & 0 \\
\hline IL-10 & ND & ND & ND & ND & $1.33 \pm 0.33$ & $1.0 \pm 0.57$ & 0 & 0 \\
\hline IL-10 + PX & ND & ND & ND & ND & 3 & $1.67 \pm 0.33$ & $2.33 \pm 0.33$ & 2 \\
\hline
\end{tabular}

Various strains of female 6- to 12-week-old mice $(n=3)$ were subjected to H\&E staining followed by histopathological analysis for degree of immune cell infiltrate (Infil.), edema (Ed.), crypt loss (CL), and goblet cell depletion (GD) as described in Methods. The first 4 strains of mice were all generated from breeding involving our starting TLR5KO. IL-10-deficient mice, untreated or treated with piroxicam (PX) for 2 weeks and given 2 weeks to recover as previously described, were obtained commercially and maintained in the same room. T4/5DKO, TLR4/5DKO.

specific immunostain ( $\alpha-$ GR-1) revealed large areas of neutrophils in the proximal colons of TLR5KO mice that were not observed in WT mice (Figure 1E) or in TLR5KO mice that lacked gross evidence of intestinal alterations. Together, these results indicate that approximately $35 \%-40 \%$ of TLR5KO mice were developing some degree of spontaneous colitis. In contrast to the cecum and colon, small intestines of TLR5KO mice appeared similar, both macroscopically and histopathologically, to those of WT littermates and did not exhibit a discernible difference in the number, size, or appearance of organized lymphoid structures in these tissues (Supplemental Figure 3).

To facilitate our understanding of how loss of TLR5 might result in spontaneous colitis, we sought to define a serum marker that would allow us to select mice that had not yet developed robust colitis. We observed that levels of serum amyloid A (SAA), a general inflammatory acute phase marker that has been shown to correlate with degree of intestinal inflammation in humans and mice $(13,14)$, could be used to identify mice with robust colitis. Specifically, whereas all WT mice assayed had SAA levels lower than $40 \mu \mathrm{g} / \mathrm{ml}, 41 \%$ (29/70) of TLR5KO mice displayed SAA levels of over $50 \mu \mathrm{g} / \mathrm{ml}$ (Figure 2A). Mice with elevated SAA also displayed elevated levels of serum lipocalin 2 (Figure 2B), which is another general marker of inflammation whose serum levels are elevated in colitis (15). SAA was most elevated in mice that had developed rectal prolapse, with every prolapsed mouse having an SAA concentration higher than $2000 \mu \mathrm{g} / \mathrm{ml}$. Further, TLR5KO mice with SAA levels greater than $100 \mu \mathrm{g} / \mathrm{ml}$ displayed gross clear evidence of colitis by the above-defined parameters whereas there were only modest differences in these parameters between WT mice and TLR5KO mice with low SAA levels (T5KO-L) (Figure 2, C-E). SAA levels also correlated with the degree of colitis determined by histopathologic assessment. Specifically, whereas histopathologic scoring showed only modest differences between WT and T5KO-L mice, TLR5KO mice with high SAA levels (T5KO-H) had colitis somewhat similar to that exhibited by IL-10KO mice, particularly IL-10KO mice that were treated with piroxicam, which promotes their development of colitis (16; Table 1). Further consistent with the colitic state, T5KO-H mice also had a significant degree of anemia and leukocytosis (Table 2), while T5KO-L mice did not differ significantly from WT littermates. Thus, SAA levels can be used to stratify TLR5KO mice as likely having or not having robust colitis. When measured shortly after weaning (25 days), 0 of 28 TLR5KO mice had elevated SAA. Rather, SAA became elevated in some mice between 5 and 8 weeks of age while some mice maintained low SAA even at 6 months. Thus, while some T5KO-L mice may ultimately develop colitis, the designation also reflects a degree of disease heterogeneity exhibited by these mice.

TLR5KO mice have an increased burden of commensal bacteria. We next considered some potential mechanisms that might underlie the colitis exhibited by TLR5KO mice. Intestinal barrier dysfunction is associated with and suggested to underlie some cases of inflammatory bowel disease in humans and the spontaneous colitis exhibited by Mdr-null mice (17). Thus, we measured epithelial barrier function by orally gavaging mice with commonly used permeability markers (HRP and FITC-dextran) and measuring their levels in the serum. We observed that robustly colitic TLR5KO mice (i.e., T5KO-H) exhibited increased intestinal permeability to these macromolecules (Figure 3). This approach did not reveal a difference in permeability between T5KO-L mice and WT littermates. Thus, increased gut permeability to macromolecules occurs in TLR5KO colitis but may not be an early event in the disease process.

Henceforth, we focused our studies on noncolitic TLR5KO mice in the hope of observing events that might drive their tendency to develop colitis. We considered whether loss of TLR5 might make mice incapable of maintaining proper homeostasis of their intestinal microflora. To begin to address this possibility, we quantitated the levels of gut bacteria that were able to grow on nonselective agar in ambient conditions. We observed that T5KO-L mice exhib-

\section{Table 2}

T5KO-H mice exhibit anemia and leukocytosis

\begin{tabular}{lccc}
\hline Assay & WT & T5KO-L & T5KO-H \\
Erythrocytes $(\mathrm{M} / \mu \mathrm{l})$ & $10.6 \pm 0.15$ & $10.3 \pm 0.19$ & $8.2 \pm 0.42^{\mathrm{A}}$ \\
Hemoglobin $(\mathrm{g} / \mathrm{dl})$ & $16.4 \pm 0.29$ & $15.8 \pm 0.20$ & $12.1 \pm 0.79^{\mathrm{A}}$ \\
Hematocrit $(\%)$ & $49.5 \pm 1.04$ & $47.1 \pm 0.55$ & $38.6 \pm 2.34^{\mathrm{A}}$ \\
White blood cells $\left(10^{3} / \mu \mathrm{l}\right)$ & $9.73 \pm 0.35$ & $11.9 \pm 0.37$ & $13.0 \pm 0.36^{\mathrm{A}}$ \\
Neutrophils $\left(10^{3} / \mu \mathrm{l}\right)$ & $0.77 \pm 0.13$ & $1.12 \pm 0.18$ & $1.65 \pm 0.33^{\mathrm{A}}$ \\
Lymphocytes $\left(10^{3} / \mu \mathrm{l}\right)$ & $8.73 \pm 0.44$ & $10.6 \pm 0.21$ & $11.7 \pm 0.23^{\mathrm{A}}$
\end{tabular}

Peripheral blood from T5KO-L, T5KO-H, or WT littermates $(n=5$ mice per condition) was subjected to standard hematologic analysis. Values are mean \pm SEM. AStatistically different from WT mice. Student's $t$ test; $P<0.05, n=5$. Monocytes, basophils, and eosinophils were not significantly different among the groups and were within the expected range. 

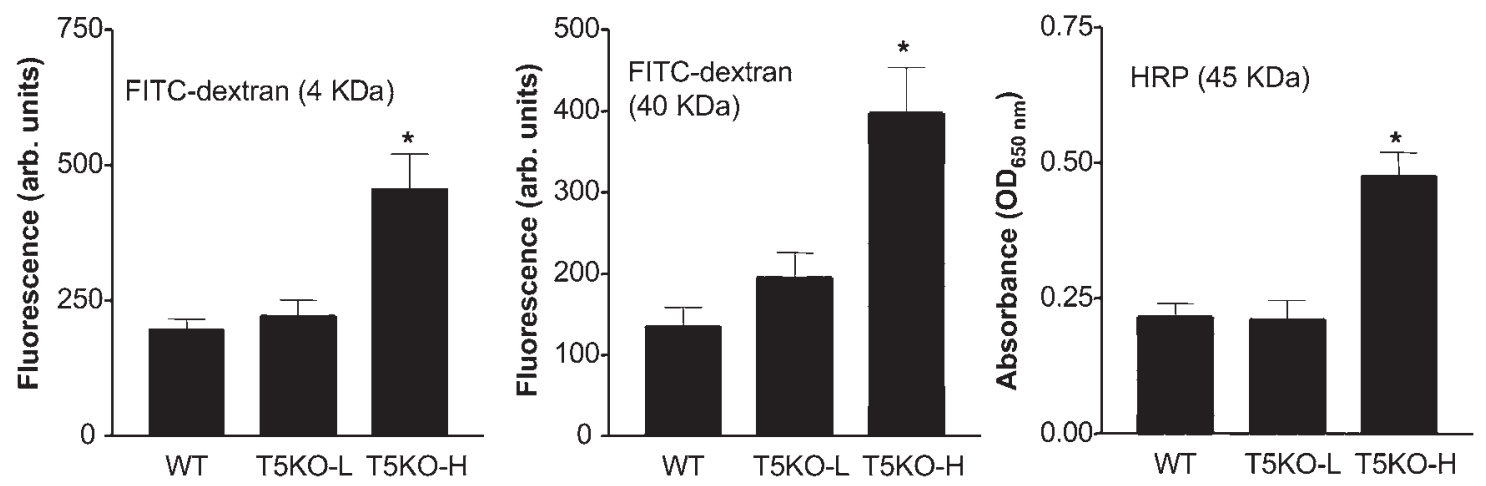

Figure 3

T5KO-H mice have increased permeability to macromolecules. T5KO-L mice (noncolitic), T5KO-H mice (colitic), and WT littermates were fasted for 3 hours and then given FITC-dextran (4 kDa or $40 \mathrm{kDa}$ ) and HRP intragastrically as described in Methods. Three hours later, mice were bled, and serum concentrations of FITC and HRP were determined by fluorimetry and spectroscopy. ${ }^{*} P<0.05$, significant difference from WT mice.

ited moderately elevated (about 5-fold) numbers of total culturable bacteria in their feces (Figure 4A). The degree of elevation was more pronounced in $\mathrm{T} 5 \mathrm{KO}-\mathrm{H}$ mice although this group also exhibited greater variability (by geometric mean, level of total culturable fecal CFUs in T5KO-H mice was $8.5 \pm 5.5$-fold higher than in T5KO-L mice). Next, we quantitated levels of bacteria in colonic segments from which we had first removed nonadherent material (as described in Methods). We observed markedly higher levels of such tightly adherent bacteria in colons of T5KO-L mice (Figure 4B). Furthermore, a number of T5KO-L mice but not their WT littermates had a modest but nonetheless clearly detectable bacterial burden in both their livers and spleens of approximately 100-200 bacteria per organ (Figure 4, C and D). Because the vast majority of intestinal bacteria are strict anaerobes and quite difficult, if not impossible, to culture, these results should be viewed as only a small sampling of the gut microflora. Nonetheless, they suggest loss of TLR5 results in significant alterations in the gut microflora.

We next sought to determine whether the bacteria we cultured from the liver/spleen of T5KO-L mice might be known pathogens or whether they were simply normal gut bacteria that may have translocated from the gut. We noted that the colonies of bacteria cultured from these organs appeared either small and whitish-yel- low or large and golden. Colonies of similar appearance could be found in the fecal cultures of both WT and T5KO-L mice although the vast majority of fecal CFUs produce much smaller, paler colonies. Thus, we hypothesized that the fecal CFUs that formed such colonies may be the same species as the CFUs we cultured from the liver and spleen. To investigate this possibility, we selected colonies of each type from the feces or organs and examined their appearance by gram staining. All were gram-positive cocci, and colony appearance correlated with their clustering style. Next, we cloned and sequenced the 16S RNAs from a small number of these isolates. Of the $6 \mathrm{~T} 5 \mathrm{KO}-\mathrm{L}$ liver/spleen isolates that were sequenced, 4 were Staphylococcus saprophyticus and 2 were Staphylococcus hominis. Of the 2 WT fecal CFU isolates that were sequenced, 1 was S. saprophyticus and 1 was S. hominis. Identifying these strains in the feces of WT mice is consistent with the knowledge that these bacteria are normal components of the intestinal microflora of cattle, rodents, and humans (18). While identifying these few CFUs as nonpathogenic does not, of course, rule out the possible presence of a pathogen in our colony, that immunodeficient mice (RAG-1KO and MyD88KO) that have shared cages and bred with these TLR5KO mice have not developed any clinical indicators of disease argues against a typical pathogen. Furthermore, the failure of the WT littermates of
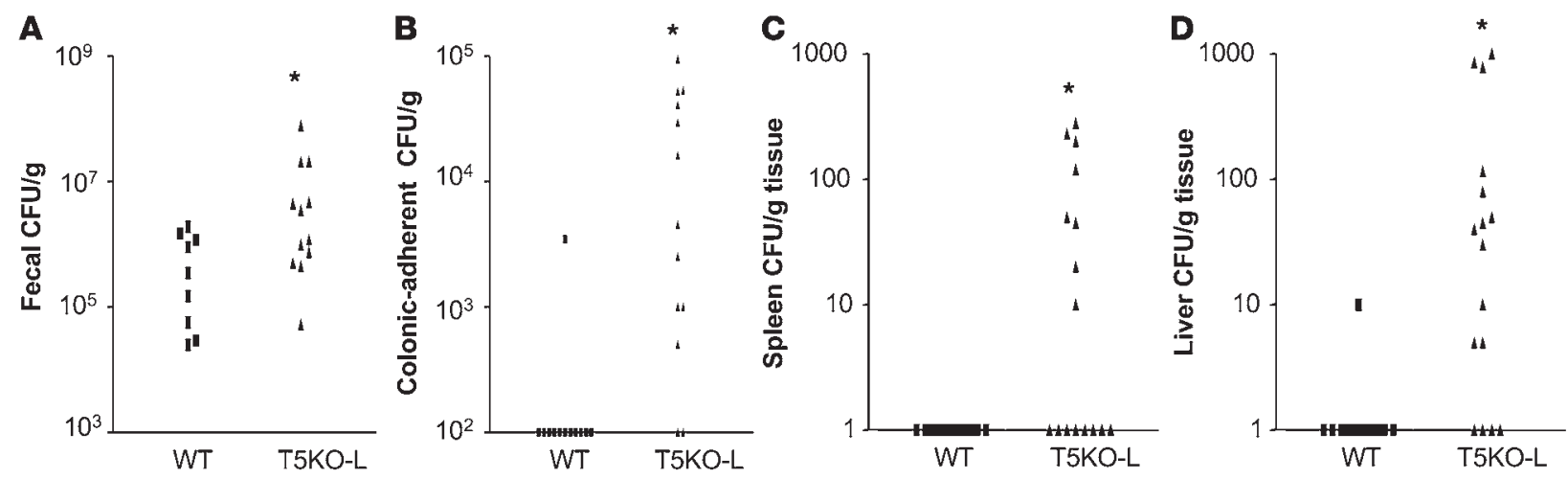

Figure 4

T5KO-L mice have an increased bacterial burden. Feces or indicated tissue from T5KO-L (i.e., not robustly colitic) or WT littermates was homogenized and ambiently cultured on nonselective media. CFUs were quantitated as described in Methods. (A) Feces; (B) washed colons; (C) spleen; (D) liver. ${ }^{*} P<0.05$, significant difference from WT mice. 
A
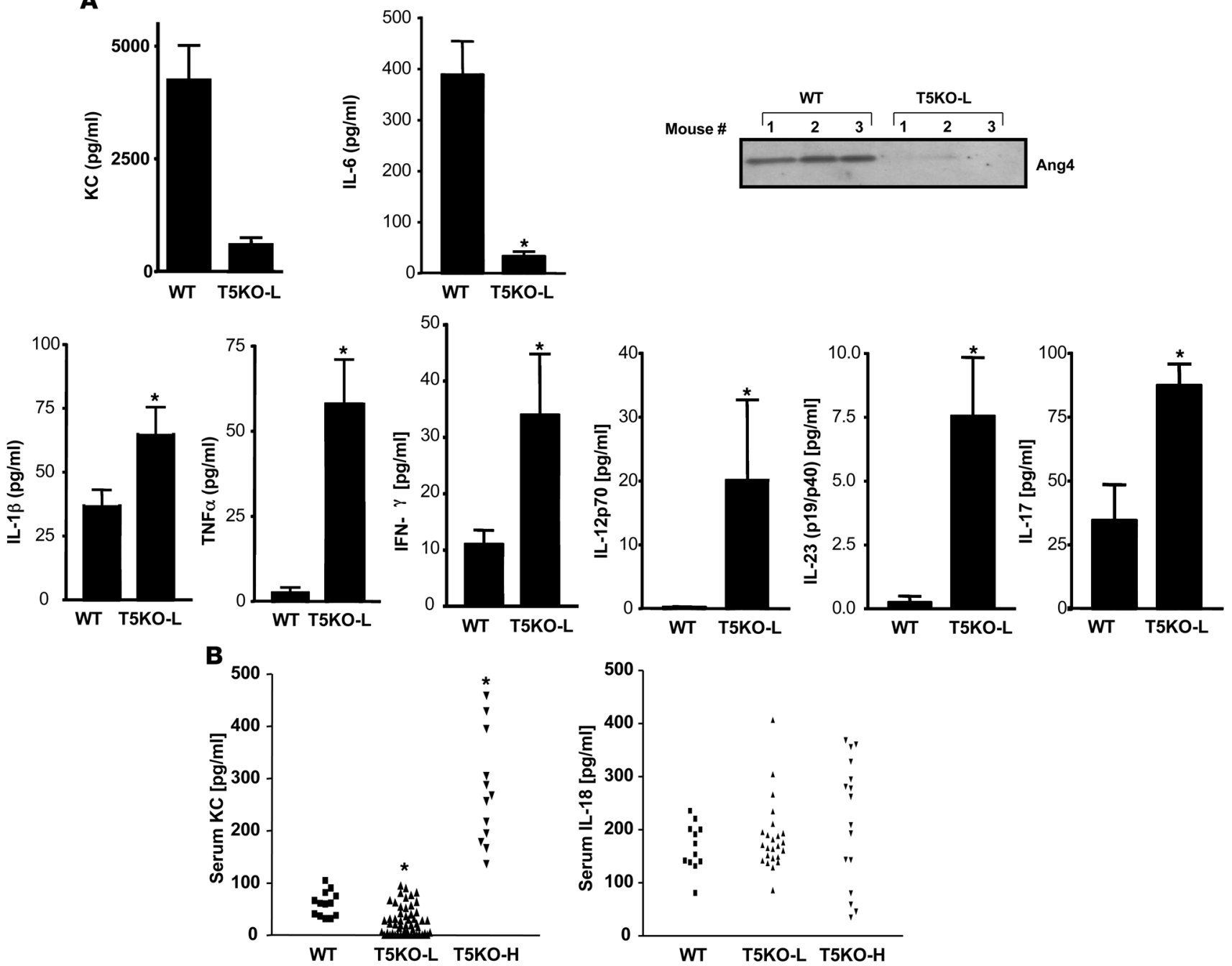

Figure 5

T5KO-L mice show altered spontaneous colonic cytokine expression. (A) Colons from WT or T5KO-L mice were cultured ex vivo for 24 hours in antibiotic-supplemented serum-free media. (A) Cytokines released into media measured by ELISA or SDS-PAGE immunoblotting (Ang4). ELISAs are shown as mean \pm SEM of at least 8 mice per condition. (B) Sera from TLR5KO mice with low or high SAA (as defined in Figure 2) or WT littermates were assayed for cytokines as in $\mathbf{A}$, but only KC and IL-18 were consistently detected. ${ }^{\star} P<0.05$, significant difference from WT mice.

T5KO to exhibit indications of colitis at 3 levels of interbreeding/ backcrossing suggests that the gut bacteria in these mice are most likely commensal microbes and argues against the presence of a truly pathogenic microbe in our colony. Thus, while Staphylococci attaining an extraintestinal localization is likely a consequence of the loss of TLR5 signaling, neither these isolates nor other bacteria colonizing these mice are pathogenic in WT mice.

TLR5KO colons exhibit dysregulated gene expression. We reasoned that reduced expression of host defense genes due to the loss of TLR5 signaling might underlie the increased bacterial burden of T5KO-L mice, although, conversely, it also seemed quite possible that the increased bacterial burden might result in greater expression of host defense genes due to increased activation of other innate immune signaling pathways. To investigate these possibilities, we used a recently described protocol (1) to assess the ongoing intestinal production of cytokines by 24-hour ex vivo culture of colonic intestinal segments. We measured cytokines that are potently induced by flagellin (KC, IL-6) as well as cytokines that are not substantially induced (at least in our hands) by purified flagellin (IFN- $\gamma$, IL-12p70, IL-23, IL-1 $\beta$, and TNF- $\alpha$ ) but which are potently upregulated in response to the TLR4 ligand LPS. We observed that, despite their increased bacterial burden, T5KO-L mice made markedly lower levels of the flagellin-induced cytokines $\mathrm{KC}$ and IL-6 (Figure 5A). In contrast, despite lack of histopathological evidence of inflammation, T5KO-L mice colons released markedly higher levels of proinflammatory cytokines (IL-1 $\beta$ and TNF- $\alpha$ ), $\mathrm{Th}_{1}$ cytokines (IFN- $\gamma$ and IL-12p70), and Th $\mathrm{h}_{17}$ cytokines (IL-17 and IL-23). We also assayed these colonic supernatants for levels of the protein angiogenin-4 (Ang4), which is thought to play an important role in host defense against gram-positive bacteria (19). Ang4 expression was markedly reduced in the colons of T5KO-L mice compared with WT littermates. Last, we also measured the levels of these cytokines in the serum of these mice. While most of these cytokines were undetectable in serum of T5KO-L mice (i.e., not 
Table 3

Summary of cDNA microarray analysis

\begin{tabular}{|c|c|c|c|c|}
\hline \multirow[b]{2}{*}{ Gene category } & \multicolumn{2}{|c|}{ T5KO-L/WT } & \multicolumn{2}{|c|}{ T5KO-H/WT } \\
\hline & $\begin{array}{l}\text { No. genes } \\
\text { elevated }\end{array}$ & $\begin{array}{c}\text { Mean } \\
\text { induction }\end{array}$ & $\begin{array}{l}\text { No. genes } \\
\text { elevated }\end{array}$ & $\begin{array}{c}\text { Mean } \\
\text { induction }\end{array}$ \\
\hline Proinflammatory cytokines & 23 & 5.2 & 45 & 8.9 \\
\hline Antimicrobials & 19 & 2.5 & 40 & 5.6 \\
\hline Cytokine receptors & 21 & 3.8 & 33 & 4.5 \\
\hline Gut hormones & 21 & 2.7 & 30 & 3.2 \\
\hline Growth and differentiation & 16 & 3.5 & 30 & 3.2 \\
\hline Cluster differentiation & 12 & 3.3 & 27 & 3.6 \\
\hline Chemokines/receptors & 9 & 2.8 & 23 & 5.0 \\
\hline Proteases & 16 & 3.6 & 22 & 4.0 \\
\hline Adhesion molecules & 5 & 6.0 & 22 & 5.8 \\
\hline Prostaglandin related & 9 & 2.5 & 21 & 5.8 \\
\hline Amino acid metabolism & 12 & 2.4 & 21 & 3.1 \\
\hline TNF related & 12 & 2.8 & 20 & 3.4 \\
\hline Lymphocyte activation & 8 & 2.6 & 19 & 3.8 \\
\hline Apoptosis (pro and anti) & 14 & 2.6 & 18 & 3.5 \\
\hline C-type lectin receptors & 5 & 1.8 & 16 & 4.3 \\
\hline Interferon related & 5 & 2.0 & 16 & 5.3 \\
\hline Stress induced & 6 & 2.9 & 14 & 5.5 \\
\hline Transcription factors & 4 & 2.3 & 13 & 2.5 \\
\hline Matrix metalloproteases & 3 & 2.5 & 12 & 3.9 \\
\hline Xenobiotic metabolism & 6 & 2.6 & 11 & 2.9 \\
\hline Epithelial barrier & 7 & 3.9 & 11 & 6.2 \\
\hline NF- $\kappa B$ signaling & 5 & 2.0 & 11 & 4.2 \\
\hline Neutrophil activation & 4 & 2.9 & 11 & 2.9 \\
\hline Antioxidant enzymes & 7 & 3.4 & 9 & 3.8 \\
\hline Water transport & 5 & 4.6 & 8 & 4.3 \\
\hline Acute-phase response & 3 & 3.4 & 7 & 8.2 \\
\hline Iron metabolism & 2 & 4.2 & 7 & 7.7 \\
\hline Reactive oxidant generation & 3 & 2.9 & 7 & 2.9 \\
\hline Complement & 3 & 2.1 & 7 & 6.4 \\
\hline Hematopoietic development & 2 & 2.2 & 6 & 2.6 \\
\hline Macrophage activation & 4 & 3.3 & 6 & 6.3 \\
\hline Protease inhibitors & 2 & 2.9 & 5 & 13.2 \\
\hline
\end{tabular}

Total colonic mRNA was pooled from groups of indicated mice $(n=3)$ and subjected to cDNA microarray analysis as described in Methods. Table lists number of genes in each functional category upregulated at least 1.8-fold relative to WT mice. Complete list of significantly upregulated genes is shown in Supplemental Table 1.

elevation of more than 800 (of 35,000 ) genes assayed. While the overall profile of these genes might most simply be described as "proinflammatory," they can also be reasonably classified into functional categories (Table 3; specific genes are listed in Supplemental Table 1) including antibacterial genes and cytokines/chemokines. Qualitatively, a similar pattern of colonic gene expression was observed in T5KO-L mice although there was a reduction both in the number of genes in each category that were significantly elevated and the extent to which their expression was elevated. The increased expression of a number of these mRNAs agreed with our protein assays of colon cultures (performed on at least 5 mice per group). Subsequently, such samples were assayed for several other proteins (IL-10, IL-18, MUC2, MMP7, MMP9), which were all upregulated in the colons of TLR5KO mice in accordance with our microarray analysis (Supplemental Figure 4). This microarray analysis also indicated that TLR5KO mice had increased expression of genes involved in LPS signaling, for example, TLR4, LPS-binding protein, and CD14 (Supplemental Table 1). The upregulation of TLR4 was confirmed by quantitative RT-PCR (Supplemental Figure 4).

IL-10 functions to reduce incidence/severity of TLR5KO colitis. The above-described studies of intestinal gene expression indicate that even $\mathrm{T} 5 \mathrm{KO}$ mice that lack histopathological evidence of colitis have dysregulated intestinal gene expression. We hypothesized that endogenous antiinflammatory mechanisms may function to prevent spontaneous colitis. For example, expression of the antiinflammatory cytokine IL-10 is elevated 2-fold in noncolitic T5KO-L and elevated 8-fold in colitic T5KO-H mice (Supplemental Figure 4 and Supplemental Table 1), an expression pattern that suggests it may be functioning to counterregulate the elevated proinflammatory gene expression exhibited by these mice. To investigate this possibility, TLR5KO mice were crossed with mice lacking the gene for IL-10 to generate TLR5/IL-10DKO (DKO, double KO) mice and IL-10KO littermates. IL-10-deficient littermates exhibited rectal prolapse at a rate similar to our original colony of IL-10KO (about $50 \%$ by 16 weeks), with the remainder being sufficiently healthy to

robustly colitic), these animals had lower serum KC than WT littermates while colitic T5KO-H mice had higher serum levels of this chemokine compared with WT littermates (Figure 5B). Serum IL-18 was elevated in some TLR5KO mice. Thus, loss of TLR5 resulted in reduced expression of some host defense genes, particularly Ang4 and 2 cytokines (KC and IL-6) that are robustly induced by flagellin. However, loss of TLR5 also resulted in increased expression of a panel of proinflammatory cytokines whose expression may be driven by other TLR ligands. To further assess altered gene expression in the colons in TLR5KO mice, we next performed a global assay of gene expression. Specifically, we used cDNA microarray to compare the relative gene expression in the colons of TLR5KO mice (T5KO-L or T5KO-H) with that of their WT littermates. These studies pooled mRNA isolated from 3 mice per group. Such analysis may reflect changes in gene expression in resident cells in the intestine and/or reflect infiltration of leukocytes, particularly in the case of the overtly colitic mice. Compared with their WT littermates, robustly colitic T5KO-H mice exhibited a significant produce offspring. In contrast, deletion of TLR5 on an IL-10-null background resulted in $80 \%$ rectal prolapse by 10 weeks (100\% by 6 months) and a uniform failure to breed (Figure 6A). Furthermore, T5/IL-10DKO mice showed a striking elevation of colonic IL-17 production whereas colitic IL-10KO mice expressed much less IL-17 than colitic T5KO mice (Figure 6B). These results suggest that the IL-10 antiinflammatory pathway plays an important role in counterregulating the proinflammatory pathways that result from loss of TLR5 but can only prevent colitis in a portion of these mice. Furthermore, the high amount of IL-17 made by colitic TLR5KO relative to colitic IL-10KO mice suggests a potential role for the $T h_{17}$ pathway in TLR5KO colitis.

TLR5KO colitis is driven by activation of TLR4. TLR5 signaling is thought to be entirely MyD88 dependent, and thus, MyD88KO mice are completely deficient in TLR5 function. However, MyD88KO mice do not develop spontaneous colitis (even when sharing cages with TLR5KO mice in our facility). This suggests that the increased bacterial burden of TLR5KO mice may result in coli- 

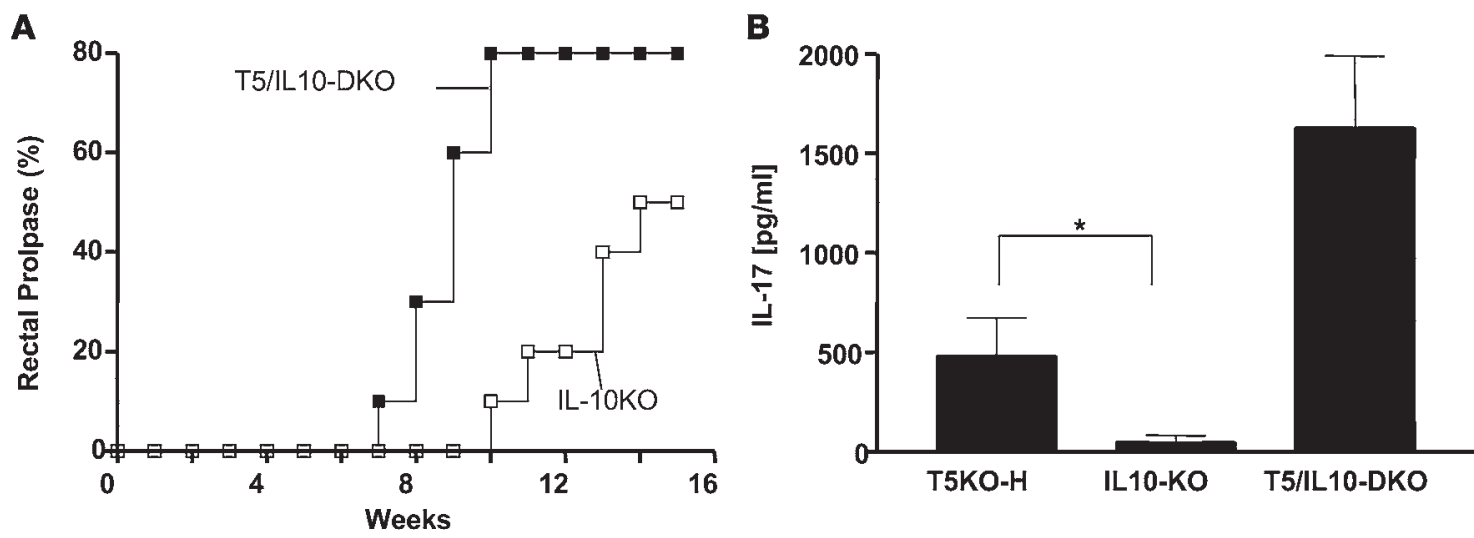

\section{Figure 6}

TLR5/IL-10DKO mice show high colonic expression of IL-17 and severe colitis. (A) TLR5/IL-10DKO mice and IL-10KO littermates were followed for incidence of rectal prolapse. Mice were euthanized upon developing prolapse, which always correlated with clear gross evidence of colitis. (B) Colons from indicated rectally prolapsed mice were isolated within 24 hours of prolapse and cultured in vitro for 24 hours; IL-17 production was measured by ELISA. * $P<0.05$.

tis via activation of other TLRs - a notion also suggested by our microarray data. In support of this possibility, colon cultures of MyD88KO mice lacked all cytokines measured, including KC, TNF- $\alpha$, and IL-1 $\beta$ (data not shown). Thus, we generated mice lacking both TLR4 and TLR5 and examined their intestinal phenotype. In contrast to TLR5KO mice, TLR4/5DKO mice neither developed rectal prolapse ( 0 of 75 - of which many were followed up to 10 months of age) nor showed detectable intestinal bleeding. Further, microscopic examination of the cecum and colon revealed only slight histopathologic abnormalities in the cecum (Table 1 and Figure 7, A and B). TLR4/5DKO mice had only a slight increase in colon weight and lacked the reduction in cecum weight and splenomegaly (and enlarged intestinal lymph nodes) that was prominent in TLR5KO mice (Figure 7C). Myeloperoxidase (MPO) levels were moderately elevated in the cecum but not in the colon (Figure 7B), and some TLR4/5DKO mice (7 of 25) had significantly elevated levels of SAA (Figure 7C). Such lack of evidence of colitis was observed in TLR4/5DKO mice at 12 weeks and 6 months of age. We also determined whether TLR4/5DKO mice had the elevated bacterial burdens that are exhibited by TLR5KO mice. We observed that, like TLR5KO mice, TLR4/5DKO mice had a significantly elevated level of culturable gut bacteria both in their feces and adherent to their colons (Figure 7D). However, unlike TLR5KO mice, TLR4/5DKO mice lacked detectable bacteria in the liver/spleen, suggesting that translocation of intestinal bacteria to these organs may occur consequent to colonic inflammation. We next compared spontaneous cytokine secretion from the colons of these mice (Figure 8). In order to both allow direct comparison with littermates and evaluate results relative to the above data, in Figure 8, we maintained the same $y$ axis scale as utilized in Figure 7. Despite an elevated intestinal bacterial load, colons from TLR4/5DKO mice exhibited less spontaneous secretion of KC, IL-6, and TNF- $\alpha$ and, like WT mice, did not secrete detectable levels of IL-12p70 or IL-23. Furthermore, TLR4/5DKO mice had markedly lower levels of serum $\mathrm{KC}$. To further evaluate the role of TLR5 in gut cytokine production, we also measured colonic cytokine production in mice lacking TLR4 (offspring of littermates of TLR4/5DKO). We observed that loss of TLR5 on a TLR4-null background still lowered colonic production of $\mathrm{KC}$ and IL- 6 but resulted in only a modest increase in TNF- $\alpha$ and IL-17. TLR4/5DKO mice had a level of IL-1 $\beta$ (Figure 8) secretion that was still elevated relative to WT littermates, suggesting this may be driven by another pathway. Last, although consistently reduced in TLR5KO mice, Ang4 expression was variable in TLR4/5DKO mice (data not shown), suggesting the possibility that this gene's expression may be regulated by multiple pathways and/or that its reduction in TLR5KO may in fact result from the cytokine/inflammatory environment.

As an additional approach, we sought to lower levels of TLR ligands via reducing levels of enteric bacteria by treating mice with antibiotics. Specifically, we next treated 5-week-old colitic TLR5KO mice for 3 weeks with streptomycin and determined whether it ameliorated their colitis. Such treatment dramatically lowered their SAA levels (Figure 9) and reduced robust inflammation and rectal prolapse (prevented prolapse in 5 of 7 treated mice). In contrast, untreated colitic TLR5KO mice uniformly (18 of 18) developed rectal prolapse within this time frame when not treated with antibiotics. Furthermore, we similarly treated TLR5KO mice that had already developed rectal prolapse and found that such treated mice had intestines and spleens of relatively normal size and appearance although such treatment did not reverse prolapse (data not shown). Together, these results suggest that loss of TLR5 results in inability to manage gut microflora, resulting in increased activation of TLR4 and likely other pathways that mediate the proinflammatory gene expression that drives recruitment of inflammatory cells, which allows intestinal bacteria to reach the spleen.

To further determine whether loss of TLR5 results in elevated responses through TLR4, we measured LPS-induced responses ex vivo of splenocytes and peritoneal macrophages. As compared with WT littermates, splenocytes from noncolitic (i.e., T5KO-L) TLR5KO mice exhibited a 3-fold higher level of IFN- $\gamma$ production in response to LPS (Supplemental Figure 5A). In contrast, macrophages isolated from WT and TLR5KO mice responded similarly to LPS (Supplemental Figure 5B), indicating that elevated response of TLR5KO splenocytes to LPS is likely acquired and may manifest in the intestine due to the altered gut bacterial homeostasis rather than reflect an inherent role of TLR5 in regulating TLR4. 


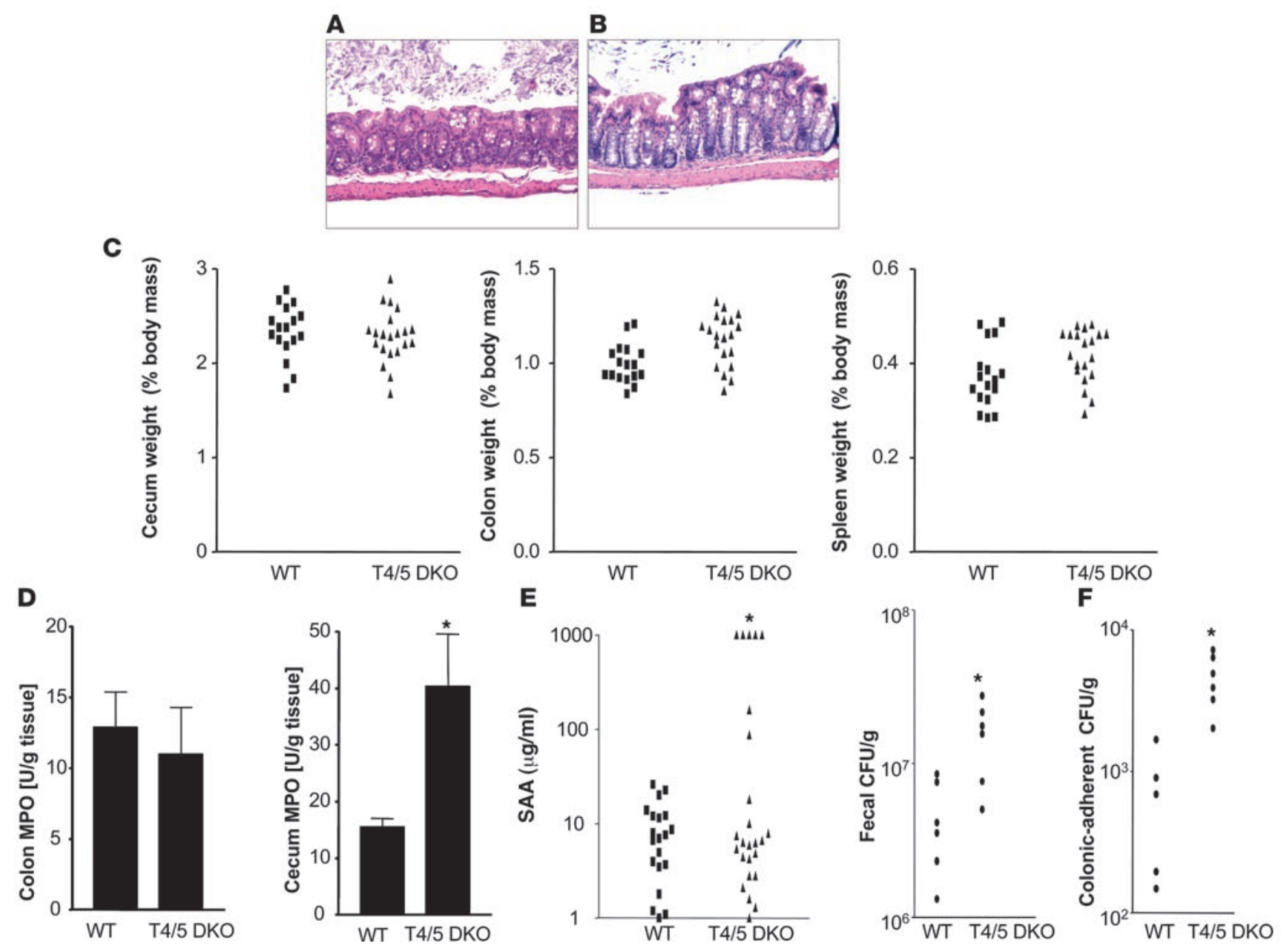

Figure 7

Deletion of TLR4 ablates colitis in TLR5KO mice. Twelve-week-old TLR4/5DKO and WT littermates were euthanized and parameters assayed. H\&E-stained (A) cecum and (B) colon. (C) Weights of the cecum, colon, and spleen. (D) Cecum and colonic MPO levels. (E) SAA levels. (F) Fecal and colon-adherent CFUs. ${ }^{*} P<0.05$.

\section{Discussion}

Based on extensive modeling of bacterial-epithelial interactions in vitro, in which flagellin is a dominant innate immune activator of proinflammatory gene expression, we hypothesized that mice lacking TLR5 would exhibit a reduced tendency to develop mucosal inflammation. In contrast, we observed that mice engineered to lack TLR5 exhibited spontaneous elevations in proinflammatory gene expression and, in a subset of mice, spontaneous colitis. Loss of TLR5 also resulted in alterations in enteric microflora. Both the proinflammatory gene expression and the spontaneous colitis that resulted from loss of TLR5 but not elevations in microflora required TLR4. Together, these observations suggest that TLR5KO colitis may originate from altered gut-microbial homeostasis that resulted from loss of TLR5. These observations indicate that TLR5 plays an essential and nonredundant role in protecting the intestine. In contrast, mice engineered to lack other individual TLRs have not generally exhibited a strong basal intestinal phenotype.

While only a portion of TLR5KO mice displayed clear clinical and/or histopathologic evidence of colitis, noncolitic TLR5KO mice still displayed clear basal abnormalities due to loss of TLR5 signaling. Specifically, noncolitic TLR5KO mice had elevated bacterial burdens in the colon and had a detectable level of such bacteria in their liver/spleens. One possibility is that loss of TLR5 may result in an increased bacterial burden in the colon via reduced intestinal expression of genes such as Ang4 that have direct antibacterial activity, although the maintenance of Ang4 expression in TLR4/5DKO mice argues against this possibility. Thus, another possibility is that the reduced colonic expression of IL-8 (KC) and IL-6, which play important roles in regulating mucosal immunity, may also eventuate in alterations in the gut microflora. Specifically, we envisage that localized epithelial bursts of IL-8 secretion may allow for limited targeted recruitment of small numbers of neutrophils that can efficiently clear bacteria that breach the epithelium. Such clearance of "microinfections" could occur without clinical manifestations and might be quite difficult to observe histopathologically. In support of this general possibility, we note that healthy mouse colon (numerous strains/sources) secreted abundant KC despite the relative rarity of neutrophils in healthy colonic mucosa. In this scenario, loss of TLR5-mediated KC production would result in inefficient clearance of these microinfections, leading to larger infections that would then trigger proinflammatory gene expres- 
A
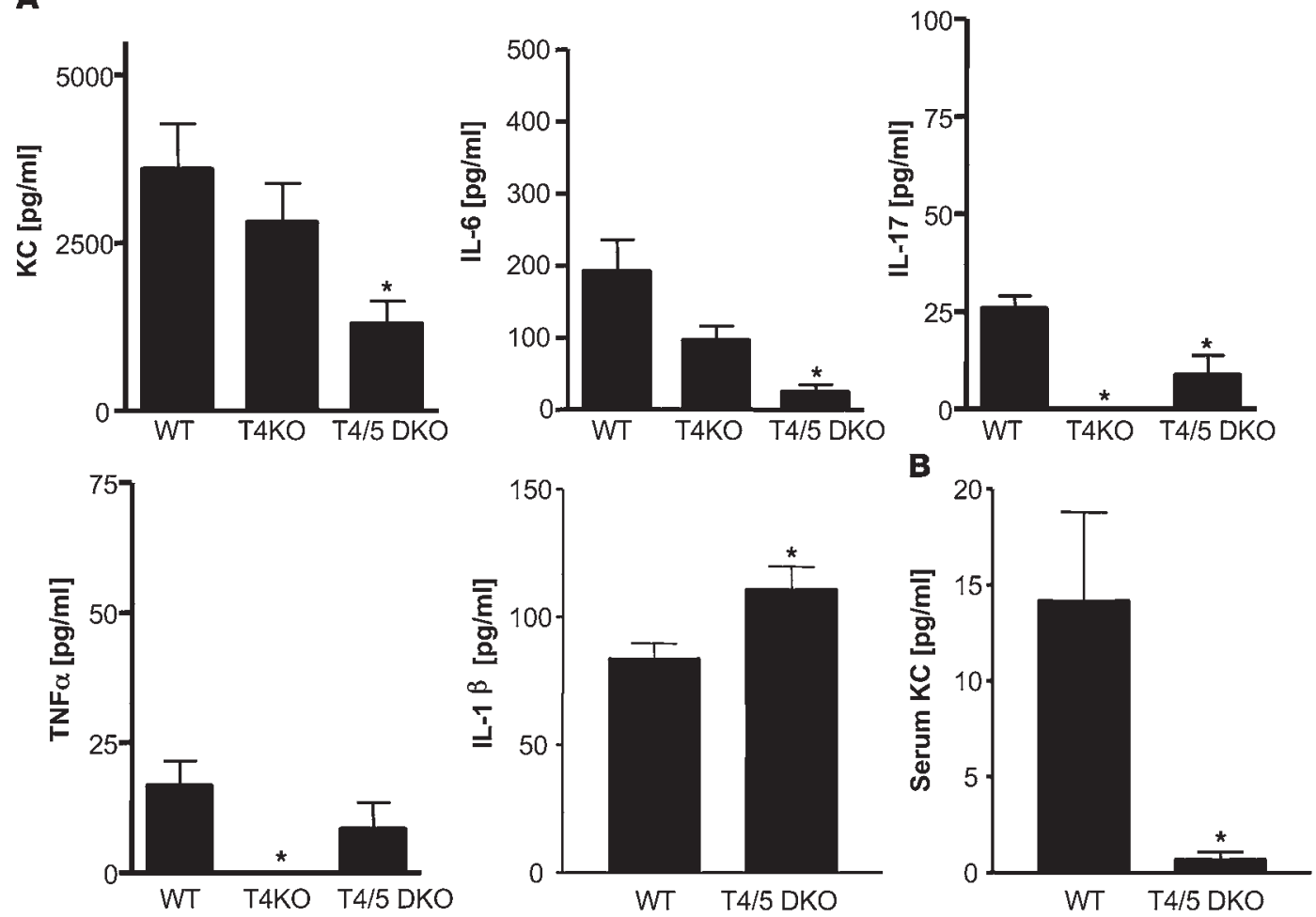

Figure 8

Deletion of TLR4 reduces elevated colonic cytokine production by TLR5KO mice. (A and B) Colons from TLR4KO, TLR4/5DKO, or WT littermates were cultured ex vivo for 24 hours in antibiotic-supplemented serum-free media. (A) Cytokines released into media measured by ELISA. (B) Serum was assayed for KC. ${ }^{*} P<0.05$, indicates significant difference from WT mice.

sion by other pathways, such as TLR4. In the case of IL-6, we speculate that, given the important role of IL- 6 in regulating adaptive immunity, reduced intestinal IL-6 may result in suboptimal function of the adaptive mucosal immune system.

Importantly, all of these TLR5-regulated host defense mechanisms can broadly help control levels of a variety of bacterial species regardless of whether a given particular species expresses flagellin. In accordance with this possibility, the Staphylococci strains that were isolated from the colon, liver, and spleen in TLR5KO mice are nonmotile and thus probably do not produce flagellin. While these Staphylococci are known opportunistic pathogens (18), their translocation to the liver and spleen did not occur in TLR4/ $5 \mathrm{DKO}$ mice, suggesting that this event is likely a consequence of disease rather than an underlying mechanism. Thus, the specific nature of the microflora that may drive TLR5KO colitis remains unclear. While our experimental approach in which TLR5KO mice were always compared directly with their WT littermates (or firstgeneration offspring) argues against a true pathogen in the colony, it does not exclude the possibility of an opportunistic pathogen that causes disease in mice deficient in TLR5 signaling. Other spontaneously colitic mice (e.g., IL-10, Mdr) are known to have disease severity vary greatly depending upon the conditions under which they are housed, with the prevailing microflora thought to be the single most important determinant. A particularly important role has been described for Helicobacter species, which are not considered pathogenic and/or monitored by most mouse facilities because they do not cause disease in WT mice. Our colony of mice (both TLR5KO and WT) tested positive for Helicobacter (PCR-based test performed on feces sent to the University of Missouri, Columbia, Missouri, USA). We speculate that differences in microflora may underlie the fact that TLR5KO mice maintained in Japan have not developed colitis severe enough to cause rectal prolapse.

Although not specifically experimentally addressed in this study, a number of observations suggest that inability of TLR5KO to maintain proper homeostasis of gut flora is largely due to loss of TLR5 signaling in intestinal epithelial cells. Namely, the reduction in colonic protein expression we observed was limited only to genes that are induced by purified flagellin and that are expressed by epithelial cells, whereas "master" inflammatory cytokines (TNF- $\alpha$, IL-1 $\beta$ ), Th $h_{1}$ cytokines (IL-12p70, IFN- $\gamma$ ), and $\mathrm{Th}_{17}$ cytokines (IL-17, IL-23), whose expression was elevated in TLR5KO mice, are neither significantly induced by flagellin nor made by epithelial cells. In accordance, epithelial cell lines are universally responsive to flagellin, and TLR5-mediated cytokine expression at another mucosal site, namely the lung, requires epithelial cells (11). Last, the notion that the altered microbial homeostasis in TLR5KO mice results from loss of TLR5 function in the epithelium is also supported by the recent findings that blockade of NF- $\kappa \mathrm{B}$ activation specifically in epithelial cells results in spontaneous colitis via a pathway dependent on TLR signaling (20) and another recent report that suggested epithelial NF-KB activation may be protective while immune cell NF- $\kappa B$ drives colitis (21). In contrast, the general unresponsiveness of epithelial cells to a number of other TLR ligands suggests that the TLR4 activation that drives colitis in TLR5KO mice may be largely mediated by hematopoietic cells, although a role for proinflammatory cytokine-induced epithelial TLR4 is possible. 
A

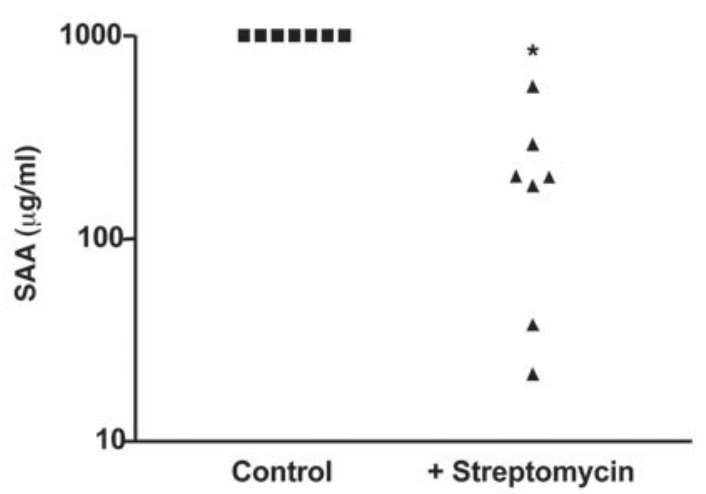

B

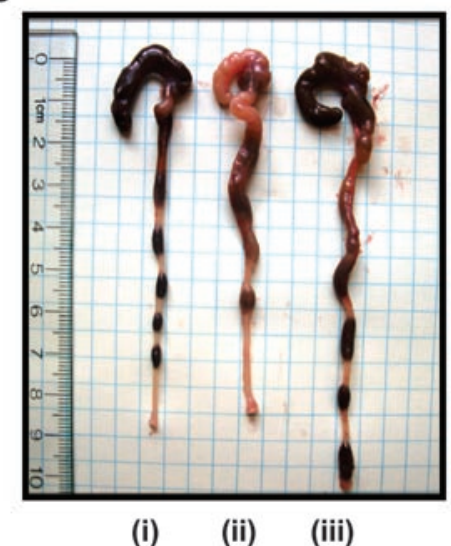

\section{Figure 9}

Antibiotic treatment ameliorates colitis in TLR5KO mice. Five-week-old colitic T5KO-H mice were treated with streptomycin (1.25\% in drinking water) for 3 weeks. (A) SAA levels before and after streptomycin treatment. (B) Representative image of gross appearance of cecum and colon. (i) Noncolitic (T5KO-L). (ii) Untreated colitic (T5KO-H). (iii) T5KO-H mice whose high SAA levels were lowered following 3 weeks of treatment with streptomycin.
The lack of robust colitis in TLR4/5DKO mice demonstrates the importance of TLR4 in driving intestinal inflammation in TLR5KO mice. The absence of spontaneous colitis and concomitant absence of colonic proinflammatory cytokines in MyD88KO mice is consistent with the possibility that TLR signaling in general is a predominant means of regulating gut inflammation. However, because MyD88KO mice are also unable to respond to IL-1 $\beta$ and IL-18 produced by Nod-like receptor pathways, it is possible that loss of TLR5 results in increased recognition of intracellular flagellin via Ipaf and/or Naip5, which function in large part by regulating production of mature IL-18 and IL-1 $\beta$ (22), which drive colitis. Additionally, increased bacterial loads may ultimately result in bacterial products such as $\mathrm{N}$-formylated peptides directly recruiting immune cells.

In addition to TLR5KO mice being potentially deficient in flagellin-induced expression of cytokines and antibacterial proteins, we speculate that TLR5KO mice may also have altered levels of apoptosis/cell survival. Such alterations could result from their increased bacterial burden or, given the increasingly appreciated role of TLR5 signaling in regulating cell survival, might result from lack of TLR5 signaling from basal levels of flagellin in the intestine. In vitro, flagellin activates both pro- and antiapoptotic signaling pathways (23), but the marked increase in apoptosis seen in vivo upon occurrence of gastroenteritis induced by flagellin-deficient Salmonella suggests a potentially important in vivo role for TLR5 signaling in promoting cell survival during pathogen challenge (24). The basolateral polarization of TLR5 and concomitant lack of proinflammatory signaling to apical flagellin in polarized epithelial cells and colons suggest that TLR5 would not be robustly activated by luminal commensal microbes $(25,26)$. However, it is possible that a low level of TLR5 signaling that might occur under such conditions is not sufficient to result in inflammation but may be sufficient to modulate homeostasis/cell survival pathways.

While complete loss of TLR5 signaling in mice results in the development of "spontaneous" colitis, the 75\% loss of TLR5 function that occurs in humans carrying a dominant negative TLR5 allele, referred to as TLR5 ${ }^{\text {STOP }}$, does not appear to closely mimic this phenotype. Specifically, carriage of TLR5 ${ }^{\text {STOP }}$ did not affect acquisition or clinical course of typhoid fever in an area where Salmonella typhi is endemic (27), nor did carriage of TLR5 $5^{\text {STOP }}$ appear to increase one's tendency to develop incidence of idiopathic intestinal inflammation, namely Crohn disease and ulcerative colitis. Rather, in some ethnic backgrounds, carriage of TLR5 $5^{\text {STOP }}$ is negatively associated with Crohn disease, suggesting that reduced TLR5 function can, in fact, protect against developing this disorder (9). While the mechanism underlying this apparent paradox is not clear, our observations still provide mechanistic insight into Crohn disease. Specifically, mutations in Nod2 have been proposed to make Nod2 nonfunctional (28) or hyperactive (29) or to result in dysregulated signaling through other TLRs (30) and thus result in Crohn disease due to, respectively, innate immune deficiency, innate immune hyperactivity, or immune dysregulation. Thus, our observation that an engineered innate immune deficiency can result in colitis supports the former possibility, namely that an innate immune deficiency due to genetic alterations in Nod2 and/or other genes might, in part, underlie Crohn disease.

\section{Methods}

Mice. Heterozygous TLR5 mice (generated in mixed C57BL/6 $\times 129 / \mathrm{Sv}$ background and backcrossed 3 generations to C57BL/6) were sent from the Research Institute for Microbial Diseases to Charles River Laboratories, where they underwent a relatively standard quarantine period in which sentinel mice were checked for most known murine pathogens. Following an additional period of quarantine/testing at Emory University, heterozygous TLR5 mice were bred to generate TLR5KO mice and WT littermates at N4, N6, and N8 levels of backcrossing. Data in this paper were generated from WT littermates and TLR5 and TLR5KO mice at N6 level. TLR5KO mice were crossed, respectively, with IL-10KO or TLR4KO mice (purchased from Jackson Laboratories) to generate TLR5/ IL-10DKO and IL-10KO littermates or TLR4/5DKO mice and WT littermates. MyD88KO (backcrossed more than 10 generations) had been bred in our facility for over 3 years and compared with true C57BL/6 mice that were originally purchased from Jackson Laboratories. All animal experiments were approved by the Emory University ethical committee.

Assessment of basal phenotype of TLR5 KO mice. Age- and sex-matched (8 to 12 week old) WT and TLR5KO mice were bled retro-orbitally and sacrificed by $\mathrm{CO}_{2}$ asphyxiation. Immediately, cecum and colon were flushed with icecold PBS, weighed (cecum was weighed before washing), and observed for spontaneous inflammation, shrinkage, and swelling in the colon. Tissues were then snap-frozen quickly in liquid nitrogen for biochemical analysis or RNA isolation or snap-frozen in OCT or fixed in $10 \%$ buffered formalin for histologic analysis. Additionally, liver and spleen were weighed. Serum was analyzed for SAA (ELISA kit from Biosource), lipocalin 2 (via Western blotting using antibody from R\&D systems), and IL-6, KC, IFN- $\gamma$, IL-1 $\beta$, and TNF- $\alpha$ using Duoset kits, IL-18 (Quantikine) from R\&D systems, 
IL-12p70, IL-17, IL-23 (eBiosciences), and IL-10 (BD Biosciences). Fecal blood and cecal/colonic MPO were measured as previously described (31). Immunofluorescent staining of neutrophils was performed on $6-\mu \mathrm{m}$ frozen sections. In brief, sections were fixed in cold acetone, washed in PBS, incubated with blocking buffer followed by $\alpha$-GR1-APC (BD - Pharmingen), washed, and mounted in media containing DAPI. APC signal was pseudocolored red. CFU levels in freshly isolated feces, liver, or spleen were determined via homogenization of material in PBS/0.01\% Triton X-100 followed by serial dilution plating on nonselective Luria-Bertani agar. To assess colonic submucosal CFUs, longitudinally cut colon was extensively washed and gently scraped with forceps, followed by homogenization/plating as above. Complete peripheral blood counts were determined by standard hematological analysis at the Missouri University Research Animal Diagnostic Laboratory (Columbia, Missouri, USA).

Histopathologic scoring of colitis. H\&E-stained sections of cecum and colon were scored for colitis as follows: cellular infiltrate $(0$, indistinguishable from typical C57BL/6 mice; 1 , mild; 2 , moderate; 3 , robust, including neutrophils); edema ( 0 , none; 1 , slight; 3 , moderate); crypt loss ( 0 , none; 1 , slight; 2 , moderate; 3 , substantial); and goblet cell depletion ( 0 , none; 1 , modest; 2 , substantial)

Colon culture. Colon pieces were cultured as described by Rakoff-Nahoum et al. (1). In brief, $1-\mathrm{cm}$ segments of proximal colons were washed in HBSS supplemented with penicillin and streptomycin (Cellgro). These segments were cultured in 24-well flat-bottom culture plates (Costar) in serum-free RPMI 1640 medium (Cellgro) supplemented with penicillin and streptomycin, L-glutamine, and nonessential amino acids. After 24 hours, supernatant was collected and centrifuged at $13,000 \mathrm{~g}$ for 10 minutes at $4^{\circ} \mathrm{C}$ and stored at $-20^{\circ} \mathrm{C}$ until analyzed. Ang 4 (rabbit polyclonal antibody; gift from Lora Hooper, University of Texas Southwest Medical Center, Dallas, Texas, USA), MUC2 (Biomeda), MMP9, and MMP7 (Chemicon) were analyzed in colon culture supernatants by immunoblotting.

In vivo epithelial barrier permeability. In vivo assay of barrier function was performed using an FITC-labeled dextran method, as described (32). In brief, 6- to 8-week-old WT and TLR5KO mice were deprived of food and water for 4 hours, and mice were gavaged with permeability tracer FITClabeled dextran $4 \mathrm{kDa}$ or $40 \mathrm{kDa}(0.6 \mathrm{mg} / \mathrm{g}$ body weight $)$ or $\mathrm{HRP}(0.02 \mathrm{mg} / \mathrm{g}$ body weight; Sigma-Aldrich). Blood was collected retro-orbitally after 3 hours, fluorescence intensity of each sample was measured (excitation, $490 \mathrm{~nm}$; emission, $520 \mathrm{~nm}$; HITACHI F-4500 Fluorescence Spectrophotometer) in serum, and FITC-dextran concentrations were determined from standard curves generated by serial dilution of FITC-dextran. HRP in the serum was measured colorimetrically using the HRP substrate trimethylbenzidine and measuring absorbance at $650 \mathrm{~nm}$.

Antibiotic treatment. Five-week-old colitic T5KO-H mice were given $1.25 \%$ streptomycin (Sigma-Aldrich) in regular drinking water for 3 weeks. Mice were then bled retro-orbitally for SAA analysis, euthanized, and analyzed for gross intestinal pathology.

Identification of bacteria by $16 \mathrm{~S} r D N A$ sequencing. 16S rRNA was amplified by PCR using forward ( $5^{\prime}$-AGAGTTTGATCACTGGCTCAG-3') and reverse (5'-TACGGCTTACCTTGTTACGACTT-3') primers and purified PCR product (QIAquick purification kit from QIAGEN) and cloned into p-GEM-T Easy vector (Promega). Subcloning Efficiency DH5 $\alpha$ Chemically Competent Cells (Invitrogen) were used for the transformation. Transformants for inserts were screened on Luria-Bertani/ampicillin/IPTG/X-Gal plates and selected positive white colonies and grown overnight at $37^{\circ} \mathrm{C}$ by shaking; plasmid DNA was isolated (QIAGEN) and sequenced using M13 forward and reverse primers at Genomic Services, Agencourt Bioscience Corp.

Microarray analysis. In brief, total RNA from colons (pooled from 3 mice per group) was prepared using TRIzOL reagent (Invitrogen) and DNase1 treated using DNaseI-Free RNase1 (QIAGEN). The integrity of RNA transcripts was verified by gel electrophoresis. Labeled cDNA was synthesized from $30 \mu \mathrm{g}$ of total RNA. Colonic RNA and mouse reference RNA (Stratagene) samples were labeled with Cy5- and Cy3-coupled dCTP (GE Healthcare), respectively. After labeling, cDNAs were purified, concentrated, and hybridized overnight with Mouse MEEBO 38K Array chip (Vanderbilt Microarray Shared Resource). Arrays were scanned with GenePix 4100A Scanner (Molecular Devices). All experiments were compared with the same Stratagene mouse reference RNA to allow the relative expression level of each gene to be compared across all experiments. After normalization, genes with intensity over 2 times of background mean were selected.

Statistics. Statistical significance was assessed by Student's $t$ test, with $P$ values of less than 0.05 considered significant.

\section{Acknowledgments}

This work was supported by grants from the Broad Medical Research Foundation, the Crohn's and Colitis Foundation of America, and the NIH (DK061417) to A.T. Gewirtz. M. Vijay-Kumar is a recipient of a research fellowship from the Crohn's and Colitis Foundation of America. We also acknowledge NIH Digestive Disease Research and Development Center grants to Emory University (DK064399). We thank Rheinallt Jones for helpful discussion and Sean Lyons and Daniel Moore III for technical assistance.

Received for publication June 25, 2007, and accepted in revised form September 12, 2007.

Address correspondence to: Andrew T. Gewirtz, Department of Pathology, Emory University, 105H Whitehead Building, 615 Michael Street, Atlanta, Georgia 30322, USA. Phone: (404) 712-9885; Fax: (404) 727-8538; E-mail: agewirt@emory.edu.
1. Rakoff-Nahoum, S., Paglino, J., Eslami-Varzaneh, F., Edberg, S., and Medzhitov, R. 2004. Recognition of commensal microflora by toll-like receptors is required for intestinal homeostasis. Cell. 118:229-241.

2. Cario, E., Gerken, G., and Podolsky, D.K. 2004. Tolllike receptor 2 enhances $\mathrm{ZO}$-1-associated intestinal epithelial barrier integrity via protein kinase C. Gastroenterology. 127:224-238.

3. Lee, J., et al. 2006. Maintenance of colonic homeostasis by distinctive apical TLR9 signalling in intestinal epithelial cells. Nat. Cell Biol. 8:1327-1336.

4. Rakoff-Nahoum, S., Hao, L., and Medzhitov, R. 2006. Role of toll-like receptors in spontaneous commensal-dependent colitis. Immunity. 25:319-329.

5. Gewirtz, A.T. 2003. Intestinal epithelial toll-like receptors: to protect. And serve? Curr. Pharm. Des. 9:1-5.
6. Zeng, H., et al. 2003. Flagellin is the major proinflammatory determinant of enteropathogenic Salmonella. J. Immunol. 171:3668-3674.

7. Lodes, M.J., et al. 2004. Bacterial flagellin is a dominant antigen in Crohn disease. J. Clin. Invest. 113:1296-1306. doi:10.1172/JCI200420295.

8. Gewirtz, A.T. 2006. Flag in the crossroads: flagellin modulates innate and adaptive immunity. Curr. Opin. Gastroenterol. 22:8-12.

9. Gewirtz, A.T., et al. 2006. Dominant-negative TLR5 polymorphism reduces adaptive immune response to flagellin and negatively associates with Crohn's disease. Am. J. Physiol. Gastrointest. Liver Physiol. 290:G1157-G1163.

10. Uematsu, S., et al. 2006. Detection of pathogenic intestinal bacteria by Toll-like receptor 5 on intestinal CD11c(+) lamina propria cells. Nat. Immunol. 7:868-874.
11. Feuillet, V., et al. 2006. Involvement of Toll-like receptor 5 in the recognition of flagellated bacteria. Proc. Natl. Acad. Sci. U. S. A. 103:12487-12492.

12. Kuhn, R., Lohler, J., Rennick, D., Rajewsky, K., and Muller, W. 1993. Interleukin-10-deficient mice develop chronic enterocolitis. Cell. 75:263-274.

13. Chambers, R.E., Stross, P., Barry, R.E., and Whicher, J.T. 1987. Serum amyloid A protein compared with C-reactive protein, alpha 1-antichymotrypsin and alpha 1-acid glycoprotein as a monitor of inflammatory bowel disease. Eur. J. Clin. Invest. 17:460-467.

14. de Villiers, W.J., Varilek, G.W., de Beer, F.C., Guo, J.T., and Kindy, M.S. 2000. Increased serum amyloid a levels reflect colitis severity and precede amyloid formation in IL-2 knockout mice. Cytokine. 12:1337-1347.

15. Peterson, C.G., Eklund, E., Taha, Y., Raab, Y., and Carlson, M. 2002. A new method for the quantification of neutrophil and eosinophil cationic pro- 
teins in feces: establishment of normal levels and clinical application in patients with inflammatory bowel disease. Am. J. Gastroenterol. 97:1755-1762.

16. Berg, D.J., et al. 2002. Rapid development of colitis in NSAID-treated IL-10-deficient mice. Gastroenterology. 123:1527-1542.

17. Resta-Lenert, S., Smitham, J., and Barrett, K.E. 2005. Epithelial dysfunction associated with the development of colitis in conventionally housed $\mathrm{mdr} 1 \mathrm{a}^{-/}$ mice. Am. J. Physiol. Gastrointest. Liver Physiol. 289:G153-G162.

18. Raz, R., Colodner, R., and Kunin, C.M. 2005. Who are you - Staphylococcus saprophyticus? Clin. Infect. Dis. 40:896-898.

19. Hooper, L.V., Stappenbeck, T.S., Hong, C.V., and Gordon, J.I. 2003. Angiogenins: a new class of microbicidal proteins involved in innate immunity. Nat. Immunol. 4:269-273.

20. Nenci, A., et al. 2007. Epithelial NEMO links innate immunity to chronic intestinal inflammation. Nature. 446:557-561.

21. Karrasch, T., Kim, J.S., Muhlbauer, M., Magness, S.T., and Jobin, C. 2007. Gnotobiotic IL-10 ${ }^{-/-}$;NF- kappa B(EGFP) mice reveal the critical role of TLR/NF-kappa B signaling in commensal bacteria-induced colitis. J. Immunol. 178:6522-6532.

22. Franchi, L., et al. 2006. Cytosolic flagellin requires Ipaf for activation of caspase-1 and interleukin 1 beta in salmonella-infected macrophages. Nat. Immunol. 7:576-582.

23. Zeng, H., et al. 2006. Flagellin/TLR5 responses in epithelia reveal intertwined activation of inflammatory and apoptotic pathways. Am. J. Physiol. Gas trointest. Liver Physiol. 290:G96-G108.

24. Vijay-Kumar, M., et al. 2006. Flagellin suppresses epithelial apoptosis and limits disease during enteric infection. Am. J. Pathol. 169:1686-1700.

25. Gewirtz, A.T., Navas, T.A., Lyons, S., Godowski, P.J., and Madara, J.L. 2001. Cutting edge: bacterial flagellin activates basolaterally expressed tlr5 to induce epithelial proinflammatory gene expression. J. Immunol. 167:1882-1885.

26. Rhee, S.H., et al. 2005. Pathophysiological role of Toll-like receptor 5 engagement by bacterial flagellin in colonic inflammation. Proc. Natl. Acad. Sci. U. S. A. 102:13610-13615.
27. Dunstan, S.J., et al. 2005. Host susceptibility and clinical outcomes in toll-like receptor 5-deficient patients with typhoid fever in vietnam. J. Infect. Dis. 191:1068-1071

28. Inohara, N., et al. 2003. Host recognition of bacterial muramyl dipeptide mediated through NOD2. Implications for Crohn's disease. J. Biol. Chem. 278:5509-5512.

29. Maeda, S., et al. 2005. Nod2 mutation in Crohn's disease potentiates NF-kappaB activity and IL-1beta processing. Science. 307:734-738.

30. Watanabe, T., Kitani, A., Murray, P.J., and Strober, W. 2004. NOD2 is a negative regulator of Toll-like receptor 2-mediated T helper type 1 responses. Nat. Immunol. 5:800-808.

31. Castaneda, F.E., et al. 2005. Targeted deletion of metalloproteinase 9 attenuates experimental colitis in mice: central role of epithelial-derived MMP. Gastroenterology. 129:1991-2008.

32. Furuta, G.T., et al. 2001. Hypoxia-inducible factor 1-dependent induction of intestinal trefoil factor protects barrier function during hypoxia. J. Exp. Med. 193:1027-1034. 